Pacific Journal of Mathematics

CORRECTTE GREEN FUNCTIONS AS A TOOL TO PROVE MODEL OF COMPETING AND DIFFUSING S 


\title{
APPROXIMATE GREEN FUNCTIONS AS A TOOL TO PROVE CORRECTNESS OF A FORMAL APPROXIMATION IN A MODEL OF COMPETING AND DIFFUSING SPECIES
}

\author{
Aart VAn HARTen AND Els VAder-Burger
}

\begin{abstract}
The purpose of this paper is two-fold; firstly, we shall derive some new results concerning a singular perturbation problem describing the stationary distribution of two competing and diffusing species; secondly, we want to demonstrate the power of a technique using approximate Green functions to prove the validity of a constructed formal approximation in a singular perturbation problem.
\end{abstract}

A mathematical model for the spatial distribution of two species consists of two coupled 2 nd order O.D.E. on the interval $[-1,1]$ with Neumann boundary conditions:

$$
\begin{array}{ll}
\varepsilon^{2} u^{\prime \prime}=f(x, u, v), & v^{\prime \prime}=g(x, u, v) \\
u^{\prime}(-1)=v^{\prime}(-1)=0, & u^{\prime}(1)=v^{\prime}(1)=0 .
\end{array}
$$

Here, $\varepsilon$ is a small parameter $>0$ and ' denotes derivation w.r.t. the $x$-variable.

This model (or rather its time-dependent version) has been proposed by several authors to explain the coexistence of competing and diffusing species in some subdomain and not elsewhere, cf. [4], [9], [5], [6], [10]. For certain non-linearities $f, g$ there is the possibility of a solution with a sharp transition phenomenon in the $u$-variable at an internal point $y \in(-1,1)$. The domain $(-1,1)$ is then subdivided in two subdomains $(-1, y)$ and $(y, 1)$ where $u$ behaves essentially different. At $y$ the jump in the $u$-behaviour is smoothed by an internal layer.

These results are derived by constructing an asymptotic approximation for $\varepsilon \downarrow 0$ of the solution of (1.1-2). In [4], only a first approximation without detailed information on the internal layer is used. In [9], all over the domain higher order terms are included in the construction. This is done by dealing with two separate problems on $[-1, y+\delta(\varepsilon)]$ and $[y+$ $\delta(\varepsilon), 1]$ with Dirichlet boundary conditions at the transition point. Free constants introduced in this way, such as $\delta(\varepsilon)$, are determined later on by requiring a smooth connection at the transition point. In this work a restriction has to be made about the non-linearity $g$ (see [9], assumption 
(ii)) just to ensure the solvability of each of the artificial problems on the subintervals $[-1, y+\delta]$ and $[y+\delta, 1]$. Though this assumption is sufficient for the construction, it is far from being sharp.

Once in [1], [9] a formal approximation has been obtained, its validity is proved. However, a purely proof-technical assumption has to be made, namely a small interaction between one of the species and the other. (For example: $g_{u}$ is required to be sufficiently small). Such an assumption does not play a role in the construction, nor does it have a biological background. In our opinion this was a somewhat unsatisfactory situation and at the same time it was for us a challenge motivating this work.

In this work we shall construct a global formal approximation of arbitrary algebraic order in $\varepsilon$ for the solution of (1.1-2), see §2. This is done under less restrictive conditions than in [9]. For the construction we use a method of matched asymptotic expansions (cf. [6]), in which the internal layer is dealt with in a direct way (without interval subdivision as in [9]). It appears, that the problem for the higher order terms in the internal layer exhibits a resonance phenomenon. This is due to an eigenvalue 0 in the spectrum of the linearized operator governing the local expansion, for the homogeneous equation has a non-trivial solution decaying exponentially towards $\pm \infty$. In order to be able to construct higher order terms a global solvability condition concerning terms in $[-1, y],[y, 1]$ as well as in the internal layer in a coupled way has to be satisfied. This solvability condition can nicely be interpreted in terms of the solvability of the reduced $(\varepsilon=0)$ problem. Namely, it is equivalent to requiring that the solution of the reduced problem is stable for small model-perturbations, i.e. small perturbations of the non-linearities $f$ and $g$.

Furthermore, we shall prove the validity of the constructed approximated under precisely the same conditions necessary to be able to do the construction. Smallness of the interaction of one of the species with the other is not necessary in this proof. The proof is based on linearisation at the constructed approximation and a fixed point argument. In itself this idea is rather standard, cf. [7], [8], [13], [1]. A central point in this type of proof is the analysis of the inverted, linearised operator in order to get an estimate for its norm. Several techniques have been used, such as maximum principles, a-prior estimates, variational principles, but in this case under the conditions we want to impose, neither of these methods seem to work. Of course, another method to analyse the inverse, linearised operator would be to use its Green kernel. This method is rather unpopular and for a good reason: exact Green functions are hard to obtain (cf. [12], [11]). However, it seems to have been overlooked, that instead of an exact Green 
function a sufficiently accurate, approximate Green function does the job just as well. Such an approximate Green function can be found in a constructive way, actually its construction is quite analogous to the construction of higher order terms in the formal approximation of the solution, see \$3. This explains that one can find such an approximate Green function under exactly the same conditions, which have to be introduced to make the construction of the formal approximation including higher order terms possible.

For these reasons the technique to use approximate Green functions to invert the linearised operator approximately is very powerful in our opinion: it is potentially applicable to a wide range of problems and it works in rather complicate situations.

2. Construction of a formal approximation. We shall consider the problem as given in (1.1-2) with $f$ and $g$ elements of $C^{\infty}\left([-1,1] \times \mathbf{R}^{2}\right)$ in the situation, where the reduced problem has a solution with a jump in the $u$-variable in some internal point of the interval. Thus we start the construction with:

2.1. The 0th order approximation. Let us call the internal transition point $y$ and the 0th order regular approximation at the left and right of $y U_{0}, V_{0}$ and $\bar{U}_{0}, \bar{V}_{0}$, respectively. Then we suppose:

Assumption 0:

The reduced regular problem for $U_{0}, V_{0}, y, \bar{U}_{0}$ and $\bar{V}_{0}$ :

$$
\begin{array}{ll}
f\left(x, U_{0}, V_{0}\right)=0 & f\left(x, \bar{U}_{0}, \bar{V}_{0}\right)=0 \\
V_{0}^{\prime \prime}=g\left(x, U_{0}, V_{0}\right) & \bar{V}_{0}^{\prime \prime}=g\left(x, \bar{U}_{0}, \bar{V}_{0}\right) \\
\text { for } x \in[-1, y] & \text { for } x \in[y, 1]
\end{array}
$$

with the boundary conditions

$$
V_{0}^{\prime}(-1)=0, \quad \bar{V}_{0}^{\prime}(1)=0
$$

and the continuity conditions in the transition point

$$
V_{0}(y)=\bar{V}_{0}(y), \quad V_{0}^{\prime}(y)=\bar{V}_{0}^{\prime}(y)
$$

plus an extra condition to determine the transition point namely

$$
\int_{U_{0}(y)}^{\bar{U}_{0}(y)} f\left(y, u, V_{0}(y)\right) d u=0
$$

has a solution such that $y \in(-1,1)$ and

$$
U_{0}, V_{0} \in C^{\infty}[-1, y], \quad \bar{U}_{0}, \bar{V}_{0} \in C^{\infty}[y, 1]
$$


with a jump in the u-variable at the transition point $y$ :

$$
U_{0}(y) \neq \bar{U}_{0}(y) \text {. }
$$

By rescaling the $u$ variable as $\left(u-U_{0}(y)\right) /\left(\bar{U}_{0}(y)-U_{0}(y)\right)$ and the $v$-variable as $v-V_{0}(y)$ and next denoting the transformed problem just as before in (1.1-2) we see, that it is no restriction to put

$$
U_{0}(y)=0, \quad \bar{U}_{0}(y)=1, \quad V_{0}(y)=0 .
$$

We observe, that because of (2.1.1): $f(y, 0,0)=f(y, 1,0)=0$. Furthermore, we introduce some notation:

$$
\begin{array}{ll}
F(u)=\int_{0}^{u} f(y, \bar{u}, 0) d \bar{u} & \\
f_{u}^{0}(x)=\frac{\partial f}{\partial u}\left(x, U_{0}(x), V_{0}(x)\right) & \text { for } x \in[-1, y] \\
\bar{f}_{u}^{0}(x)=\frac{\partial f}{\partial u}\left(x, \bar{U}_{0}(x), \bar{V}_{0}(x)\right) & \text { for } x \in[y, 1]
\end{array}
$$

and $g_{u}^{0}, \bar{g}_{u}^{0}$ are defined analgously.

Note, that $F(0)=0$ and that (2.1.4) implies: $F(1)=0$.

In addition to assumption 0 we require:

Assumption 0':

$$
f_{u}^{0}>0 \quad \text { on }[-1, y], \quad \bar{f}_{u}^{0}>0 \quad \text { on }[y, 1]
$$

and:

$$
F(u)>0 \text { on }(0,1) \text {. }
$$

The condition in (2.1.9) is assumed because of the construction of higher order terms, see (2.2.2). As a consequence of (2.1.10) we can calculate the transition layer in the $u$-variable near the transition point in 0th order:

$$
u \simeq T_{0}(\xi)+\cdots \quad \text { with } \xi=(x-y) / \varepsilon
$$

where $T_{0}$ satisfies

$$
\frac{d^{2} T_{0}}{d \xi^{2}}=f\left(y, T_{0}, 0\right), \quad \lim _{\xi \rightarrow-\infty} T_{0}(\xi)=0, \quad \lim _{\xi-\infty} T_{0}(\xi)=1
$$

The solution is given implicitly by

$$
T_{0}(\xi)=\tau\left(\xi-\xi_{0}\right), \quad \int_{1 / 2}^{\tau(\xi)} \frac{d u}{\sqrt{2 F(u)}}=\zeta
$$


Here $\xi_{0}$ is an undetermined constant, due to translation invariancy in (2.1.12). The study of higher order terms will reveal the value of $\xi_{0}$, as we shall see.

It is a nice exercise to show, that $T_{0}$ approaches its limit values at $\infty$ in an exponential way:

$$
\begin{array}{ll}
\tau(\zeta)=A \exp (\mu \zeta) \cdot(1+O(\exp (\mu \zeta)) & \text { for } \zeta \rightarrow-\infty \\
\frac{d^{k} \tau}{d \zeta^{k}}=A_{k}^{\prime} \exp (\mu \zeta) \cdot(1+O(\exp (\mu \zeta)) & \text { for } \zeta \rightarrow-\infty \\
\tau(\zeta)-1=B \exp (-\nu \zeta) \cdot(1+O(\exp (-\nu \zeta)) & \text { for } \zeta \rightarrow \infty \\
\frac{d^{k} \tau}{d \zeta^{k}}=B_{k}^{\prime} \exp (-\nu \zeta) \cdot(1+O(\exp (-\nu \zeta)) & \text { for } \zeta \rightarrow \infty
\end{array}
$$

with $\mu=f_{u}^{0}(y)>0, \nu=\bar{f}_{y}^{0}(y)>0$ and $k \geq 1$.

Furthermore, $T_{0}$ is strictly increasing on $(-\infty, \infty)$.

2.2. On the effect of model perturbations. As we saw, the 0th order terms in the approximation have been found with the proviso that $\xi_{0}$ still has to be determined. Our next concern will be the construction of higher order terms. For this construction we need another assumption. It is interesting, that this assumption can be understood in terms of model perturbations. Namely, suppose that the model is slightly perturbed, such that the non-linearities change into: $f+\delta f_{p}, g+\delta g_{p}$. Here $\delta$ measures the size of the perturbations and $f_{p}, g_{p} \in C^{\infty}\left([-1,1] \times \mathbf{R}^{2}\right)$. Now, we want the solution of the reduced problem (2.1-4) to be "stable" under such model perturbations, i.e. the perturbed reduced model has a solution for small $\delta$ of the form:

$$
\begin{gathered}
U_{0}+\delta u_{p}+O\left(\delta^{2}\right), \quad V_{0}+\delta v_{p}+O\left(\delta^{2}\right), \\
\bar{U}_{0}+\delta \bar{u}_{p}+O\left(\delta^{2}\right), \quad \bar{V}_{0}+\delta \bar{v}_{p}+O\left(\delta^{2}\right), \quad y+\delta y_{p}+O\left(\delta^{2}\right) .
\end{gathered}
$$

In order to make this condition more concrete we shall derive the problem for the perturbed quantities $u_{p}, v_{p}, \bar{u}_{p}, \bar{v}_{p}, y_{p}$. It is convenient to introduce the following shorthand notation

$$
\mathscr{L}(U, V, \bar{U}, \bar{V}, C)=\left(r_{1}, r_{2}, \bar{r}_{1}, \bar{r}_{2} ; \alpha, \bar{\alpha} ; j, j^{\prime} ; \beta\right)
$$

for the following problem

$$
\begin{array}{cc}
f_{u}^{0} U+f_{v}^{0} V+r_{1}=0 & \bar{f}_{u}^{0} \bar{U}+\bar{f}_{v}^{0} \bar{V}+\bar{r}_{1}=0 \\
V^{\prime \prime}=g_{u}^{0} U+g_{v}^{0} V+r_{2} & \bar{V}^{\prime \prime}=\bar{g}_{u}^{0} \bar{U}+\bar{g}_{v}^{0} \bar{V}+\bar{r}_{2} \\
U^{\prime}(-1)=\alpha, & \bar{U}^{\prime}(1)=\bar{\alpha}
\end{array}
$$




$$
\begin{aligned}
& U^{\prime}(y)=U(y)+j, \\
& \bar{U}^{\prime}(y)+\bar{V}_{0}^{\prime \prime}(y) C=U^{\prime}(y)+V_{0}^{\prime \prime}(y) C+j^{\prime} \\
& C \cdot \int_{0}^{1} f_{x}(y, u, 0) d u \\
& +\left(V(y)+C V_{0}^{\prime}(y)\right) \cdot \int_{0}^{1} f_{v}(y, u, 0) d u+\beta=0 .
\end{aligned}
$$

Then the perturbed quantities have to satisfy

$$
\mathscr{L}\left(u_{p}, v_{p}, \bar{u}_{p}, \bar{v}_{p}, y_{p}\right)=\left(f_{p}^{0}, g_{p}^{0}, \bar{f}_{p}^{0}, \bar{g}_{p}^{0} ; 0,0 ; 0,0 ; 0\right)
$$

with

$$
f_{p}^{0}=f_{p}\left(x, U_{0}(x), V_{0}(x)\right), \quad \bar{f}_{p}^{0}=f_{p}\left(x, \bar{U}_{0}(x), \bar{V}_{0}(x)\right),
$$

etc. Note, that $\mathscr{L}$ plays the role of a linearized operator of the reduced problem. Let us now formulate the condition in a precise way:

\section{Assumption 1:}

The homogeneous problem

$$
\mathscr{L}(U, V, \bar{U}, \bar{V}, C)=(0,0,0,0 ; 0,0 ; 0,0 ; 0)
$$

has only the trivial solution.

Using a Fredholm alternative it is then easily seen, that inhomogeneous problems such as (2.2.1) or (2.2.6) can be solved in a unique way. Further, it is not difficult to verify, that if the inhomogeneous term in (2.2.1) is in $\left(C^{\infty}[-1, y]\right)^{2} \times\left(C^{\infty}[y, 1]\right)^{2} \times \mathbf{R}^{5}$, the solution is an element of $\left(C^{\infty}[-1, y]\right)^{2} \times\left(C^{\infty}[y, 1]\right)^{2} \times \mathbf{R}$. Moreover, the solution of (2.2.1) depends continuously on the right hand side in an obvious way.

It will be clear, that assumption 1 implies the previously mentioned "stability" property, because of the implicit function theorem.

In $\$ 2.3$ we shall see, that inhomogeneous problems such as (2.2.1) are the heart of the construction of higher order terms.

2.3. A formal approximation of order $\varepsilon^{N+1}$. The structure of this formal approximation of the solution of (1.1-2) is as follows:

$$
\begin{aligned}
u= & Z_{1}^{n}=\left(U_{\operatorname{def}}^{N}+G^{N+1}\right) H+\left(\bar{U}^{N}+\bar{G}^{N+1}\right) \bar{H} \\
& +(1-H-\bar{H}) T^{N} \\
v \simeq & Z_{2}^{N} \underset{\operatorname{def}}{=}\left(V^{N}+K^{N+2}\right) H+\left(\bar{V}^{N}+\bar{K}^{N+2}\right) H \\
& +(1-H-\bar{H}) Y^{N+2}+\varepsilon^{N+1}\left(k_{N+1}+\bar{k}_{N+1}\right) .
\end{aligned}
$$


Here $U^{N}, V^{N}$ and $\bar{U}^{N}, \bar{V}^{N}$ denote the regular expansion at the left and right of the transition point

$$
\begin{aligned}
U^{N} & =\sum_{n=0}^{N} \varepsilon^{n} U_{n}(x) & \bar{U} & =\sum_{n=0}^{N} \varepsilon^{n} \bar{U}_{n}(x) \\
V^{N} & =\sum_{n=0}^{N} \varepsilon^{n} V_{n}(x) & \bar{V}^{N} & =\sum_{n=0}^{N} \varepsilon^{n} \bar{V}_{n}(x) .
\end{aligned}
$$

Further $G^{N+1}, K^{N+2}$ and $\bar{G}^{N+1}, \bar{K}^{N+2}$ describe boundary layer corrections at $x=-1$ and $x=1$, respectively

$$
\begin{aligned}
G^{N+1} & =\sum_{n=1}^{N+1} \varepsilon^{n} G_{n}(\eta) & \bar{G}^{N+1} & =\sum_{n=1}^{N+1} \varepsilon^{n} \bar{G}_{n}(\bar{\eta}) \\
K^{N+2} & =\sum_{n=3}^{N+2} \varepsilon^{n} K_{n}(\eta) & \bar{K}^{N+2} & =\sum_{n=3}^{N+2} \varepsilon^{n} \bar{K}_{n}(\bar{\eta})
\end{aligned}
$$

with layer variables $\eta=(x+1) / \varepsilon$ and $\bar{\eta}=(1-x) / \varepsilon$.

The other terms $T^{N}, Y^{N+2}$ describe the behaviour in the internal layer

$$
T^{N}=\sum_{n=0}^{N} \varepsilon^{n} T_{n}(\xi), \quad Y^{N+2}=\sum_{n=0}^{N+2} \varepsilon^{n} Y_{n}(\xi)
$$

where the layer variable is $\xi=(x-y) / \varepsilon$, as before in (2.1.11).

The functions $H$ and $\bar{H}$ are suitably chosen cut-off functions. We take

$$
H(x, \varepsilon)=\chi\left(\frac{y-x}{\sigma(\varepsilon)}\right), \quad \bar{H}(x, \varepsilon)=\chi\left(\frac{x-y}{\bar{\sigma}(\varepsilon)}\right)
$$

where $\chi$ is a smooth function on $\mathbf{R}$ with the properties $\chi(t) \equiv 0$ for $t \leq 1$, $\chi^{\prime} \geq 0, \chi(t) \equiv 1$ for $t \geq 2$. The order functions $\sigma(\varepsilon), \bar{\sigma}(\varepsilon)$ will be specified in \$2.3.3.

Finally, $k_{N+1}$ and $\bar{k}_{N+1}$ are introduced to take care that the approximation will exactly satisfy the boundary conditons:

$$
\begin{aligned}
& k_{N+1}=-\frac{d K_{N+2}}{d \eta}(0) \cdot(1+x) \cdot \chi\left(\frac{y-x}{4(1+y)}\right) \\
& \bar{k}_{N+1}=\frac{d \bar{K}_{N+2}}{d \bar{\eta}}(0) \cdot(x-1) \cdot \chi\left(\frac{x-y}{4(1-y)}\right) .
\end{aligned}
$$

2.3.1. On the internal layer. Substitution of (2.3.4) into (1.1-2) and collecting terms of order $\varepsilon^{n}$ provides us with the equations governing the internal layer expansion:

$$
\frac{d^{2} T_{n}}{d \xi^{2}}-\hat{f}_{u}^{0}(\xi) T_{n}=F_{n}^{1}(\xi) \text { for } n \geq 1
$$


with

$$
\begin{aligned}
\text { (i) } F_{1}^{1} & =\xi \hat{f}_{x}^{0}+Y_{1} \hat{f}_{v}^{0} \\
\text { (ii) } F_{n}^{1} & =\left(\xi \hat{f}_{x u}^{0}+T_{1} \hat{f}_{u u}^{0}+Y_{1} \hat{f}_{u v}^{0}\right) T_{n-1}+Y_{n} \hat{f}_{v}^{0}+\tilde{F}_{n}^{1}, \\
\tilde{F}_{n}^{1} & =\frac{1}{n !} \frac{d^{n}}{d \varepsilon^{n}}\left\{f\left(y+\varepsilon \xi, \sum_{k=0}^{n-2} \varepsilon^{k} T_{k}, \sum_{k=0}^{n-1} \varepsilon^{k} Y_{k}\right)\right\}_{\varepsilon=0} \\
& \text { for } n \geq 2
\end{aligned}
$$

and

$$
\frac{d^{2} Y_{n+2}}{d \xi^{2}}=F_{n}^{2}(\xi) \text { for } n+2 \geq 1
$$

with

$$
\begin{aligned}
\text { (i) } F_{-1}^{2} & =0, \quad F_{0}^{2}=\hat{g}^{0} \\
\text { (ii) } F_{n}^{2} & =T_{n} \hat{g}_{u}^{0}+\tilde{F}_{n}^{2} \\
& \tilde{F}_{n}^{2}=\frac{1}{n !}\left(\frac{d}{d \varepsilon}\right)^{n}\left\{g\left(y+\varepsilon \xi, \sum_{k=0}^{n-1} \varepsilon^{k} T_{k}, \sum_{k=0}^{n} \varepsilon^{k} Y_{k}\right)\right\}_{\varepsilon=0} \\
& \text { for } n \geq 1 .
\end{aligned}
$$

Here the superscript ${ }^{\wedge} 0$ means: evaluation with the argument $\left(y, T_{0}(\xi), 0\right)$.

Because of the matching for $\xi \rightarrow \pm \infty$ we are only interested in solutions of (2.3.1.1), which together with their derivatives are polynomially bounded. Now, $T_{0}^{\prime}$, the derivative of $T_{0}$ w.r.t. $\xi$, is a solution of the homogeneous equation corresponding to (2.3.1.1) and $T_{0}^{\prime}$ decays exponentially for $\xi \rightarrow \pm \infty$, see (2.1.14). Multiplying both sides in (2.3.1.1) with $T_{0}^{\prime}$ and integrating from $-\infty$ to $\infty$ it is clear, that this equation can only have a solution, which together with its derivatives is polynomially bounded, if:

$$
\int_{-\infty}^{\infty} F_{n}^{1}(\xi) T_{0}^{\prime}(\xi) d \xi=0
$$

On the other hand, if $F_{n}^{1}(\xi)$ and its derivatives are polynomially bounded and the orthogonality condition (2.3.1.5) is satisfied, then (2.3.1.1) has solutions of the desired type and these solutions are given by

$$
\begin{aligned}
& T_{n}=\hat{T}_{n}-A_{n} T_{0}^{\prime} \\
& \hat{T}_{n}(\xi)=T_{0}^{\prime}(\xi) \int_{0}^{\xi}\left[T_{0}^{\prime}(\bar{\xi})\right]^{-2} \int_{-\infty}^{\bar{\xi}} T_{0}^{\prime}(\tilde{\xi}) F_{n}^{1}(\tilde{\xi}) d \tilde{\xi} d \bar{\xi}
\end{aligned}
$$


with a free constant $A_{n}$, which will be determined later on. Therefore, the problem for the $T_{n}$ 's is characterised by a non-zero kernel of the operator. Thus, it turns out that the expansion of the $u$-variable in the internal layer is of resonant type.

Of course, the solution of (2.3.1.3) is given by

$$
\text { (2.3.1.7) } \quad Y_{1}=\gamma_{1}+\gamma_{1}^{\prime} \xi, \quad Y_{n}=\gamma_{n}+\gamma_{n}^{\prime} \xi+\hat{Y}_{n}(\xi), \quad \text { for } n \geq 2
$$

with $\hat{Y}_{n}(\xi)=\int_{0}^{\xi}(\xi-\bar{\xi}) F_{n-2}^{2}(\bar{\xi}) d \bar{\xi}$ and certain constants $\gamma_{n}, \gamma_{n}^{\prime}$, which will follow later on by matching with the regular expansion.

Note, that $\hat{T}_{n}, \hat{Y}_{n}$ and $\hat{Y}_{n+1}$ follow in a unique way once $T_{0}, \ldots, T_{n-1}$ and $Y_{1}, \ldots, Y_{n-1}$ have been determined.

Using induction w.r.t. $n$ it is now easy to check, that $\hat{T}_{n}$ and $\hat{Y}_{n}$ are smooth functions, which behave as a polynomial of degree $n$ plus an exponentially vanishing term both for $\xi \rightarrow-\infty$ and $\xi \rightarrow \infty$.

$$
\begin{array}{rlrl}
\text { (2.3.1.8) } & \hat{T}_{n} \underset{\xi \rightarrow-\infty}{\sim} P_{n}(\xi)=\sum_{k=0}^{n} p_{k}^{n} \xi^{k}, & \hat{Y}_{n} \underset{\xi \rightarrow-\infty}{\sim} Q_{n}(\xi)=\sum_{k=0}^{n} q_{k}^{n} \xi^{k} \\
\hat{T}_{n} \underset{\xi \rightarrow \infty}{\sim} \bar{P}_{n}(\xi)=\sum_{k=0}^{n} \bar{p}_{k}^{n} \xi^{k}, & \hat{Y}_{n} \underset{\xi \rightarrow \infty}{\sim} \bar{Q}_{n}(\xi)=\sum_{k=0}^{N} \bar{q}_{k}^{n} \xi^{k} .
\end{array}
$$

Moreover, the derivatives behave in analogous way. After these preparations we are in the position to deduce some useful relations. In the first place we shall exploit the matching of the $v$-component in the layer and the regular expansion. This leads us to certain jump conditions for the regular expansion at the free surface in the following way.

A. Comparing constants terms in the expansion of $Y^{N}$ for $\xi \rightarrow \pm \infty$ and $V^{N}, \bar{V}^{N}$ for $x \rightarrow y$ we find that we have to impose the following matching relations:

$$
\bar{V}_{1}(y)=V_{1}(y)=\gamma_{1}
$$

and for $n \geq 1$ :

$$
V_{n+1}(y)=\gamma_{n+1}+q_{0}^{n+1}, \quad \bar{V}_{n+1}(y)=\gamma_{n+1}+\bar{q}_{0}^{n+1} .
$$

Hence:

$$
\bar{V}_{n+1}(y)=V_{n+1}(y)+j_{n},
$$

where $j_{n}={ }_{\text {def }} q_{0}^{n+1}-\bar{q}_{0}^{n+1}$ is completely determined by $T_{0}, \ldots, T_{n-1}$, $Y_{1}, \ldots, Y_{n-1}$. Note, that the jump condition in (2.3.1.11) is of the form given in (2.2.4). 
B. Comparing linear terms in the expansion of $Y^{N}$ for $\xi \rightarrow \pm \infty$ with those arising by expansion of $V^{N}, \bar{V}^{N}$ in the variable $y+\varepsilon \xi$ we are led to the following matching relations:

$$
\gamma_{1}^{\prime}=V_{0}^{\prime}(y)
$$

and for $n \geq 0$ :

$$
V_{n+1}^{\prime}(y)=\gamma_{n+2}^{\prime}+q_{1}^{n+2}, \quad \bar{V}_{n+1}^{\prime}(y)=\gamma_{n+2}^{\prime}+\bar{q}_{1}^{n+2} .
$$

Hence:

$$
\bar{V}_{n+1}^{\prime}(y)=V_{n+1}^{\prime}(y)+q_{1}^{n+2}-\bar{q}_{1}^{n+2} .
$$

We shall now demonstrate, that (2.3.1.14) is of the form given in (2.2.4) where the role of $C$ is played by $\xi_{0}$ if $n=0$ and by $A_{n}$ if $n \geq 1$.

In the case $n=0$ we use that

$$
\begin{aligned}
& \frac{d \hat{Y}_{n+2}}{d \xi}=\int_{0}^{\xi} g\left(y, T_{0}(\bar{\xi}), 0\right) d \bar{\xi} \\
& \quad=g(y, 1,0) \xi+\int_{-\xi_{0}}^{\infty}[g(y, \tau(\zeta), 0)-g(y, 1,0)] d \zeta, \text { for } \xi \rightarrow \infty \\
& \quad=g(y, 0,0) \xi-\int_{-\infty}^{-\xi_{0}}[g(y, \tau(\zeta), 0)-g(y, 0,0)] d \zeta, \text { for } \xi \rightarrow-\infty
\end{aligned}
$$

with $\tau(\zeta)$ as in (2.1.13-14). As a consequence we obtain

$$
q_{1}^{2}-\bar{q}_{1}^{2}=j_{0}^{\prime}+\xi_{0}[g(y, 0,0)-g(y, 1,0)],
$$

with

$$
\begin{aligned}
j_{0}^{\prime}= & -\int_{-\infty}^{0}[g(y, \tau(\zeta), 0)-g(y, 0,0)] d \zeta \\
& -\int_{0}^{\infty}[g(y, \tau(\zeta), 0)-g(y, 1,0)] d \zeta
\end{aligned}
$$

Since $V_{0}^{\prime \prime}(y)=g(y, 0,0)$ and $\bar{V}_{0}^{\prime \prime}(y)=g(y, 1,0),(2.3 .1 .14)$ yields:

$$
\bar{V}_{1}^{\prime}(y)+\bar{V}_{0}^{\prime \prime}(y) \xi_{0}=V_{1}^{\prime}(y)+V_{0}^{\prime \prime}(y) \xi_{0}+j_{0}^{\prime} .
$$

This relation is indeed of the form given in (2.2.4) and $j_{0}^{\prime}$ is completely determined.

In the case $n \geq 1$ one has

$$
\frac{d \hat{Y}_{n+2}}{d \xi}=-A_{n} \int_{0}^{\infty} g\left(y, T_{0}(\bar{\xi}), 0\right) T_{0}^{\prime}(\bar{\xi}) d \bar{\xi}+\left(\frac{d \hat{Y}_{n+2}}{d \xi}\right)_{r}
$$


where the latter term is completely determined by $T_{0}, \ldots, T_{n-1}, Y_{1}, \ldots, Y_{n}$. It is now easy to verify that (2.3.1.14) yields:

(2.3.1.16) $\bar{V}_{n+1}^{\prime}(y)+\bar{V}_{0}^{\prime \prime}(y) A_{n}=V_{n+1}^{\prime}(y)+V_{0}^{\prime \prime}(y) A+j_{n}^{\prime}$.

This is again of the form specified in (2.2.4) and $j_{n}^{\prime}$ depends only on $T_{0}, \ldots, T_{n-1}, Y_{1}, \ldots, Y_{n}$.

Another useful relation is found by analyzing the orthogonality condition (2.3.1.5) somewhat further.

C. In the first place substitution of $(2.3 .1 .2-(\mathrm{i}))$ with

$$
Y_{1}=V_{1}(y)+V_{0}^{\prime}(y) \xi
$$

and a change of variables $\zeta=\xi+\xi_{0}$ yields

$$
\begin{aligned}
& \xi_{0} \cdot \int_{0}^{1} f_{x}(y, u, 0) d u \\
& \quad+\left(V_{1}(y)+\xi_{0} V_{0}^{\prime}(y)\right) \cdot \int_{0}^{1} f_{v}(y, u, 0) d u+\beta_{0}=0
\end{aligned}
$$

with

$$
\beta_{0}=\int_{-\infty}^{\infty}\left\{f_{x}(y, \tau(\zeta), 0)+V_{0}^{\prime}(y) f_{v}(y, \tau(\zeta), 0)\right\} \zeta \tau^{\prime}(\zeta) d \zeta
$$

Note, that (2.3.1.18) is of the form given in (2.2.5) with $\xi_{0}$ playing the role of $\mathrm{C}$. The constant $\beta_{0}$ is known.

Next, we substitute $F_{n+1}^{1}$, as in (2.3.1.2-(ii)) in the orthogonality condition for the equation $T_{n+1}$ and we use the following expressions for $T_{n}$ and $Y_{n+1}: T_{n}=\hat{T}_{n}-A_{n} T_{0}^{\prime}$ and

$$
\text { (2.3.1.19) } \quad Y_{n+1}=\left(V_{n+1}(y)+q_{0}^{n+1}\right)+\left(V_{n}^{\prime}(y)-q_{1}^{n+1}\right) \xi+\hat{Y}_{n+1} \text {. }
$$

Then several integrations by parts in combination with the equation for $T_{1}$ lead us to

$$
\begin{aligned}
& A_{n} \cdot \int_{0}^{1} f_{x}(y, u, 0) d u \\
& \quad+\left(V_{n+1}(y)+A_{n} V_{0}^{\prime}(y)\right) \cdot \int_{0}^{1} f_{v}(y, u, 0) d u+\beta_{n}=0
\end{aligned}
$$

with

$$
\begin{aligned}
\beta_{n}=\int_{-\infty}^{\infty} T_{0}^{\prime} & {\left[\hat{T}_{n}\left\{\xi \hat{f}_{x u}^{0}+Y_{1} \hat{f}_{u v}^{0}\right\}\right.} \\
& \left.+\hat{f}_{v}^{0}\left\{-q_{0}^{n+1}+\left(V_{n}^{\prime}(y)-q_{1}^{n+1}\right) \xi+\hat{Y}_{n+1}\right\}+\tilde{F}_{n+1}^{1}\right] d \xi
\end{aligned}
$$

The important thing is, that (2.3.1.20) is again of the form given in (2.2.5), while $\beta_{n}$ depends only on $T_{0}, \ldots, T_{n-1}, Y_{1}, \ldots, Y_{n}, V_{n}$. The rather surprising conclusion of this section is, that the relations derived by analysis of 
the internal layer have a lot to do with the reduced problem after a model perturbation, though no internal layer is present in that problem, only a transition point.

2.3.2. A scheme to determine all higher order terms uniquely. The construction of the higher order terms is organised as an iterative procedure. At the start $U_{0}, V_{0}, y, \bar{U}_{0}, \bar{V}_{0}$ are known and $T_{0}$ is known except for the value of $\xi_{0}$. Further, $G_{1}$ and $\bar{G}_{1}$ can now in the usual way be determined as the solutions of the homogeneous boundary layer equations vanishing at infinity and correcting the error in the b.c. due to $U_{0}, \bar{U}_{0}$.

$$
G_{1}(\eta)=\frac{U_{0}^{\prime}(-1)}{a} e^{-a \eta}, \quad \bar{G}_{1}(\bar{\eta})=\frac{-U_{0}^{\prime}(1)}{\bar{a}} e^{-\bar{a} \bar{\eta}}
$$

with

$$
a=\sqrt{f_{u}^{0}(-1)}>0, \quad \bar{a}=\sqrt{\hat{f}_{u}^{0}(1)}>0 .
$$

Then we proceed to determine the other terms and constants according to the following scheme:

step 1.

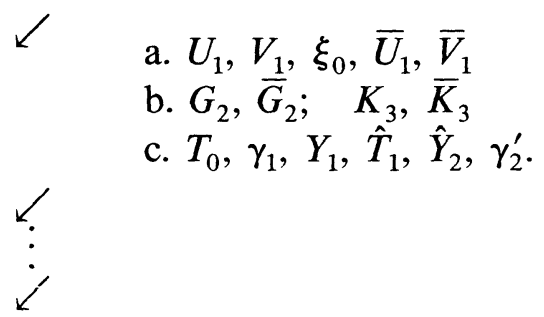

$$
\begin{aligned}
& \text { step }(n+1), n \geq 1 \text { a. } U_{n+1}, V_{n+1}, A_{n}, \bar{U}_{n+1}, \bar{V}_{n+1} \\
& \text { b. } G_{n+2}, \bar{G}_{n+2}, K_{n+3}, \bar{K}_{n+3} \\
& \text { c. } T_{n}, \gamma_{n+1}, Y_{n+1}, \hat{T}_{n+1}, \hat{Y}_{n+2}, \gamma_{n+2}^{\prime} .
\end{aligned}
$$

The problem for $U_{n+1}, V_{n+1}, A_{n}, \bar{U}_{n+1}, \bar{V}_{n+1}$ is of the form (2.2.1):

$$
\begin{aligned}
& \mathscr{L}\left(U_{n+1}, V_{n+1}, A_{n}, \bar{U}_{n+1}, \bar{V}_{n+1}\right) \\
& \quad=\left(r_{n}^{1}, r_{n}^{2}, \bar{r}_{n}^{1}, \bar{r}_{n}^{2} ; \alpha_{n}, \bar{\alpha}_{n} ; j_{n}, j_{n}^{\prime} ; \beta_{n}\right)
\end{aligned}
$$

also in the case $n=0$, if we identify $A_{0}$ with $\xi_{0}$.

Substitution of the regular expansion in (1.1-2) yields:

$$
\begin{aligned}
& r_{n}^{1}=\frac{1}{(n+1) !}\left[\left(\frac{d}{d \varepsilon}\right)^{n+1} f\left(x, \sum_{k=0}^{n} \varepsilon^{k} U_{k}, \sum_{k=0}^{n} \varepsilon^{k} V_{k}\right)\right]_{\varepsilon=0}+U_{n-1}^{\prime \prime} \\
& r_{n}^{2}=\frac{1}{(n+1) !}\left[\left(\frac{d}{d \varepsilon}\right)^{n+1} g\left(x, \sum_{k=0}^{n} \varepsilon^{k} U_{k}, \sum_{k=0}^{n} \varepsilon^{k} V_{k}\right)\right]_{\varepsilon=0},
\end{aligned}
$$

where for $n=0$ we interprete $U_{n-1}^{\prime \prime}$ as 0 . 
For $\bar{r}_{n}^{1}, \bar{r}_{n}^{2}$ analogous expressions hold.

The boundary values $\alpha_{n}, \bar{\alpha}_{n}$ are taken in such a way that the effect of $K_{n+2}, \bar{K}_{n+2}$ is annihilated

$$
\alpha_{n}=-\frac{d K_{n+2}}{d \xi}(0), \quad \bar{\alpha}_{n}=\frac{d \bar{K}_{n+2}}{d \xi}(0)
$$

In the case $n=0$ we take $\alpha_{n}=\bar{\alpha}_{n}=0$, of course. The definition of $j_{n}$ was given in (2.3.1.11), $j_{n}^{\prime}$ was introduced in (2.3.1.15) for $n=0$ and in (2.3.1.16) for $n \geq 1$. It is now clear that at the end of $n$th iteration step all information to determine $U_{n+1}, V_{n+1}, A_{n}, \bar{U}_{n+1}, \bar{V}_{n+1}$ is available and part a of the $(n+1)$ th iteration step consists of solving (2.3.2.2). This can be done because of assumption 1 .

Part b of the $(n+1)$ th iteration step is a matter of more or less standard boundary layer theory. $G_{n+2}$ is defined as the solution of:

$$
\begin{aligned}
& \frac{d^{2} G_{n+2}}{d \eta^{2}}-a^{2} G_{n+2}=w_{n+2}^{1} \\
& \frac{d G_{n+2}}{d \eta}(0)=-U_{n+1}^{\prime}(-1), \quad \lim _{\eta \rightarrow \infty} G_{n+2}(\eta)=0
\end{aligned}
$$

with

$$
\begin{array}{r}
w_{n+2}^{1}=\frac{1}{(n+2) !}\left[( \frac { d } { d \varepsilon } ) ^ { n + 2 } \left\{f\left(x, U^{n+1}+G^{n+1}, V^{n+1}+K^{n+2}\right)\right.\right. \\
\left.\left.-f\left(x, U^{n+1}, V^{n+1}\right)\right\}\right]_{\varepsilon=0}
\end{array}
$$

where $x=-1+\varepsilon \eta$ and $U^{n+1}$, etc. are defined as before in (2.3.2-3). Using induction one finds, that $G_{n+2}$ is uniquely determined and equal to a polynomial of degree $(n+1)$ in $\xi$ multiplied with $\exp (-a \xi)$. The second component is found from

$$
\frac{d^{2} K_{n+3}}{d \eta^{2}}=w_{n+1}^{2}, \quad \lim _{\eta \rightarrow \infty} K_{n+3}(\eta)=0
$$

with

$$
\begin{aligned}
& w_{n+1}^{2}=\frac{1}{(n+1) !}\left[( \frac { d } { d \varepsilon } ) ^ { n + 1 } \left\{g\left(x, U^{n+1}+G^{n+1}, V^{n+1}+K^{n+1}\right)\right.\right. \\
& \left.\left.-g\left(x, U^{n+1}, V^{n+1}\right)\right\}\right]_{\varepsilon=0}
\end{aligned}
$$


It is easy to check, that $K_{n+3}$ is uniquely determined and equal to a polynomial of degree $n$ in $\xi$ multiplied with $\exp (-a \xi)$. Of course the construction of $\bar{G}_{n+2}$ and $\bar{K}_{n+3}$ is completely analogous.

So, only the verification of part $\mathrm{c}$ of the $(n+1)$ th induction step now remains, but this is an immediate consequence of the results in \$2.3.1, namely (2.3.1.6) provides us with $T_{n}$ and $\hat{T}_{n+1},(2.3 .1 .10)$ yields $\gamma_{n+1} ; Y_{n+1}$ and $\hat{Y}_{n+1}$ follow from (2.3.1.7) and finally (2.3.1.13) combined with our knowledge of $\hat{Y}_{n+2}$ gives us the value of $\gamma_{n+2}^{\prime}$.

This completes the calculation of the formal approximation as defined in (2.3.1).

2.4. Estimation of the remainder in the equation. The result of the construction is, that the formal approximation has the following properties:

$$
\begin{aligned}
& \varepsilon^{2} \frac{d^{2} Z_{1}^{N}}{d x^{2}}=f\left(x, Z_{1}^{N}, Z_{2}^{N}\right)+R_{1}^{N}, \quad \frac{d^{2} Z_{2}^{N}}{d x^{2}}=g\left(x, Z_{1}^{N}, Z_{2}^{N}\right)+R_{2}^{N} \\
& \left(Z_{1}^{N}\right)^{\prime}(-1)=\left(Z_{2}^{N}\right)^{\prime}(-1)=0, \quad\left(Z_{1}^{N}\right)^{\prime}(1)=\left(Z_{2}^{N}\right)^{\prime}(1)=0 .
\end{aligned}
$$

Note, that the formal approximation satisfies the boundary conditions exactly, but some remainders arise in the equation. If $N$ is sufficiently large, these errors are small. In the maximum norm on $[-1,1]$ we get an estimate

$$
\left|R_{1}^{N}\right|_{\max }+\left|R_{2}^{N}\right|_{\max } \leq \tilde{R}_{N}\left[\varepsilon \ln \left(\frac{1}{\varepsilon}\right)\right]^{N+1},
$$

if the order functions $\sigma$ and $\bar{\sigma}$ in the cut-off near the transition point (see (2.3.5)) are suitably chosen:

$$
\sigma(\varepsilon)=\sigma_{0} \varepsilon \ln \left(\frac{1}{\varepsilon}\right), \quad \bar{\sigma}(\varepsilon)=\bar{\sigma}_{0} \varepsilon \ln \left(\frac{1}{\varepsilon}\right)
$$

with $\sigma_{0}$ and $\bar{\sigma}_{0}$ sufficiently large, $\varepsilon$-independent constants.

The verification of (2.4.3) consists of two elements. The first element is a piece of algebraic reasoning to show, that the internal layer expansion and the regular expansion have a good overlap in the regions $\sigma \leq y-x$ $\leq 2 \sigma$ and $\bar{\sigma} \leq x-y \leq 2 \bar{\sigma}$, compare [9], [2]. Consider on one hand $T^{N}$ for $\xi \rightarrow-\infty, T^{N} \sim \sum_{n=0}^{N} \varepsilon^{k} P_{n}(\xi)$ with $P_{n}$ as in (2.3.1.8) and on the other hand: the Taylor series up to $O\left(\varepsilon^{N}\right)$ of $\sum_{n=0}^{N} \varepsilon^{n} U_{n}(y+\varepsilon \xi)$ being $\sum_{n=0}^{N} \varepsilon^{n} \tilde{P}_{n}(\xi)$. Then both $P_{N}$ and $\tilde{P}_{n}$ are polynomials in $\xi$ of degree $n$ and using the matching it is clear that these polynomials have the same constant term and for $n \geq 1$ also the same linear term. By the same reasoning $\sum_{n=1}^{N+2} \varepsilon^{n} \check{Q}_{n}$ with $\check{Q}_{n}=Q_{n}+\gamma_{n}+\gamma_{n}^{\prime} \xi, Q_{n}$ as in (2.3.1.8) and the 
Taylor series up to $O\left(\varepsilon^{N+2}\right)$ of $\sum_{n=1}^{N+2} \varepsilon^{n} V_{n}(y+\varepsilon \xi)=\sum_{n=1}^{N+2} \varepsilon^{n} \tilde{Q}_{n}$ are such that $\check{Q}_{n}$ and $\tilde{Q}_{n}$ are polynomials in $\xi$ of degree $n$ with the same constant and linear term. Moreover, by construction both $\sum_{n=0}^{N} \varepsilon^{n} P_{n}, \sum_{n+1}^{N+2} \varepsilon^{n} \check{Q}_{n}$ and $\sum_{n=0}^{N} \varepsilon^{n} \tilde{P}_{n}, \sum_{n=0}^{N+2} \varepsilon^{n} \tilde{Q}_{n}$ satisfy equation $1.1-2$ with an error $O\left(\varepsilon^{N+1}\right)$. It is now easy to verify with just some algebra on the coefficients of the polynomials, that $P_{n}=\tilde{P}_{n}, n=0, \ldots, N$ and $\check{Q}_{n}=\tilde{Q}_{n}, n=1, \ldots, N+2$. In this way we can prove the overlap property:

$$
\begin{gathered}
\left|U^{N}-T^{N}\right| \leq C|\varepsilon \xi|^{N+1} \\
\left|V^{N}-Y^{N+2}\right| \leq C|\varepsilon \xi|^{N+3}
\end{gathered}
$$

on the regions $\sigma \leq y-x \leq 2 \sigma$ and $\bar{\sigma} \leq x-y \leq 2 \bar{\sigma}$ with an $\varepsilon$-independent constant $C$.

The second element is a rather lengthy, but in principal elementary calculation using the well-known properties of Taylor series expansion to check (2.4.3) in the various subregions of $[-1,1]$. For example, in the region $y-\sigma \leq x \leq y+\bar{\sigma}$ containing the internal layer we obtain for the error in the 2 nd component the formula

$$
\begin{aligned}
R_{2}^{N, i}= & \frac{1}{N !} \int_{0}^{1}(1-t)^{N}\left(\frac{d}{d t}\right)^{N+1} \\
& \times g\left(y+\varepsilon t \xi, \sum_{n=0}^{N}(\varepsilon t)^{n} T_{n}, \sum_{n=1}^{N+2}(\varepsilon t)^{n} Y_{n}\right) \cdot d t
\end{aligned}
$$

and it is easy to check, that

$$
\left|R_{2}^{N, i}\right| \leq C \varepsilon^{N+1}(1+|\xi|)^{N+1}
$$

on that region.

In the region $y-2 \sigma \leq x \leq y-\sigma$ one splits the remainder as

$$
\begin{aligned}
R_{2}^{N}= & R_{2}^{N, i}-\frac{d^{2}}{d x^{2}}\left\{\left(V^{N}-Y^{N+2}\right) H\right\} \\
& +g\left(X, T^{N}+\left(U^{N}-T^{N}\right) H, Y^{N+2}+\left(V^{N}-Y^{N+2}\right) H\right) \\
& -g\left(X, T^{N}, Y^{N+2}\right) .
\end{aligned}
$$

Using (2.4.6) and the overlap properties as in (2.4.5) together with analogous estimates for the derivatives, such as

$$
\left|\frac{d}{d x}\left(V^{N}-Y^{N+2}\right)\right| \leq C_{1} \varepsilon^{N+1} \xi^{N} \text { for } y-2 \sigma \leq x \leq y-\sigma
$$

we then find in this region an estimate for $R_{2}^{N}$ of the same type as (2.4.7).

Further details of the proof of (2.4.3) are left to the reader. 
3. Proof of the validity of the formal approximation. In this section we shall prove the existence of a solution $u, v$ of (1.1-2), such that

$$
\left|u-Z_{1}^{N}\right|_{\max }+\left|v-Z_{2}^{N}\right|_{\max }=O\left(\varepsilon^{N+1}\right)
$$

where ||$_{\max }$ is the maximum norm of $[-1,1]$.

The proof will be based on a contraction principle, which we shall first discuss in general.

3.1. The general idea of the proof. Suppose, that one is in the situation where for a singular perturbation problem

$$
F_{\varepsilon}(u)=0
$$

a formal approximation $Z$ has been constructed, for which

$$
F_{\varepsilon}(Z)=R
$$

where the remainder term is small, i.e. $R \in B$ with $B$ a Banach-space with norm, | |, say:

$$
|R|=\mu(\varepsilon) .
$$

Let us denote the linearised operator $D F_{\varepsilon}(Z)$ by $L_{\varepsilon}$ and the strict non-linear terms by $N_{\varepsilon}$, i.e.

$$
F_{\varepsilon}(Z+v) \underset{\text { def }}{=} F_{\varepsilon}(Z)+L_{\varepsilon} v+N_{\varepsilon}(v) .
$$

The problem for the remainder term

$$
v=u-Z
$$

can then be written as

$$
L_{\varepsilon} v=-R-N_{\varepsilon}(v) .
$$

Now, suppose that an approximate right inverse operator $\left(L_{\varepsilon}^{-1}\right)^{\prime}$ of $L_{\varepsilon}$ can be found, such that

$$
L_{\varepsilon}\left(L_{\varepsilon}^{-1}\right)^{\prime}=I+\delta(\varepsilon) K_{\varepsilon}
$$

with $I$ the identity on $B, K_{\varepsilon}$ a bounded operator from $B$ into itself and the operator norm satisfies:

$$
\left|\delta(\varepsilon) K_{\varepsilon}\right| \leq \frac{1}{2} \quad \text { for } 0<\varepsilon \leq \varepsilon_{1} .
$$

Let us put

$$
v=\left(L_{\varepsilon}^{-1}\right)^{\prime} w
$$

then $w$ has to satisfy:

$$
\left(I+\delta K_{\varepsilon}\right) w=-R-N_{\varepsilon}\left(\left(L_{\varepsilon}^{-1}\right)^{\prime} w\right) .
$$

This is an equation which can easily be solved by a fixed point argument. 
Suppose the non-linearity satisfies a Lipschitz condition

$$
\left|N_{\varepsilon}\left(\left(L_{\varepsilon}^{-1}\right)^{\prime} w_{1}\right)+N_{\varepsilon}\left(\left(L_{\varepsilon}^{-1}\right)^{\prime} w_{2}\right)\right| \leq l(\varepsilon, \rho)\left|w_{1}-w_{2}\right|
$$

for all $w_{1}, w_{2} \in B(\rho)=\{w \in B|| w \mid \leq \rho\}$ with a Lipschitz constant

$$
l(\varepsilon, \rho)=\frac{\rho}{\nu(\varepsilon)} \text { for } 0<\rho \leq \rho_{2}(\varepsilon), 0<\varepsilon \leq \varepsilon_{2}
$$

with $\nu(\varepsilon)>0$.

If the remainder is sufficiently small

$$
4 \mu(\varepsilon) \leq \rho_{0}=\min \left\{\rho_{2}(\varepsilon), \frac{1}{16} \nu(\varepsilon)\right\}
$$

for $0<\varepsilon \leq \varepsilon_{0} \leq \min \left(\varepsilon_{1}, \varepsilon_{2}\right)$, then there is a unique solution $w$ of (3.1.10) in $B\left(\rho_{0}\right)$. This solution satisfies the estimate

$$
|w| \leq 4 \mu(\varepsilon) .
$$

In terms of a solution of (3.1.1) this means, that if

$$
|r| \leq \frac{1}{4} \rho_{0}
$$

then there is a unique solution of (3.1.1) in the set $Z+\left(L_{\varepsilon}^{-1}\right)^{\prime} B\left(\rho_{0}\right)$ and the following error estimate holds

$$
|u-Z| \leq 4\left|\left(L_{\varepsilon}^{-1}\right)^{\prime}\right| \cdot|R| .
$$

In comparison with the proofs in [7], [8] or [13] the new point is that in this approach one works with an approximate right inverse of the linearised operator. In cases where $L$ is a (partial or ordinary) differential operator (or an integro-differential operator, etc.) one can try to find such approximate inverses by using an approximate function of Green satisfying

$$
L_{\varepsilon} \operatorname{Gr}(x, t)=\delta(x-t)+h
$$

where $h$ is $o(1)$ for $\varepsilon \downarrow 0$ in a suitable sense. Of course, for systems of differential equations we can proceed analogously using an approximate Green matrix. The operator $\left(L_{\varepsilon}^{-1}\right)^{\prime}$ defined by

$$
\left[\left(L_{\varepsilon}^{-1}\right)^{\prime} u\right](x)=\int_{\Omega} \operatorname{Gr}(x, t) u(t) d t
$$

with $\Omega$ the domain of the functions under consideration is then a good candidate to be used as an approximate inverse with the desired properties.

3.2. Construction of an approximate Green matrix. In our case $L_{\varepsilon}$ is an ordinary differential operator with as its domain the pairs of $C^{2}$ functions 
on $[-1,1]$ satisfying homogeneous boundary conditions of Neumann type:

$$
L_{\varepsilon}=\left(\begin{array}{cc}
\varepsilon^{2} \frac{d^{2}}{d x^{2}} & 0 \\
0 & 1
\end{array}\right)-\left(\begin{array}{cc}
\check{f}_{u} & \check{f}_{v} \\
\check{g}_{u} & \check{g}_{u}
\end{array}\right) .
$$

Here ${ }^{\vee}$ means evaluation with the argument $\left(x, Z_{1}^{N}, Z_{2}^{N}\right)$. We shall now construct an approximate Green matrix

$$
\mathrm{Gr}=\left(\begin{array}{ll}
I^{1} & J^{1} \\
I^{2} & J^{2}
\end{array}\right)
$$

where $\operatorname{Gr}(x, t)$ has the following properties:

(i) $\mathrm{Gr} \in C\left([-1,1]^{2}\right)$ and the restrictions of $\mathrm{Gr}$ to the triangles $\Delta_{1}=$ $\{(x, t) \mid-1 \leq x \leq t \leq 1\}$ and $\Delta_{2}=\{(x, t) \mid-1 \leq t \leq x \leq 1\}$ are smooth up to the boundary of these triangles.

(ii) The jump in the derivative $\partial \mathrm{Gr} / \partial x$ at $x=t,[\partial \mathrm{Gr} / \partial x]_{x=t}$, is of the following form

$$
\left[\frac{\partial \mathrm{Gr}}{\partial x}\right]_{x=t}=1+\varepsilon \mathbf{A}(x, \varepsilon)
$$

with 1 the identity and $\mathbf{A}$ uniformly bounded on $[-1,1]$ for $\varepsilon \downarrow 0$.

(iii) The matrix function " $L_{\varepsilon} \mathrm{Gr}$ ", defined as $\left(L_{\varepsilon} \mathrm{Gr}\right)(x, t)$ for $x \neq t$ with a jump at $x=t$, but without the distributional terms for $x=t$ in $L_{\varepsilon} \mathrm{Gr}$, satisfies:

$$
\int_{-1}^{1}\left\|“ L_{\varepsilon} \operatorname{Gr}(x, t) "\right\| d t \leq K \varepsilon^{\nu}
$$

with constants $K, \nu>0$ independent of $x$ and $\varepsilon$ and \|\| some $\varepsilon$-independent matrix norm.

(iv) Gr satisfies the homogeneous Neumann boundary conditions

$$
\left.\frac{\partial \mathrm{Gr}}{\partial x}\right|_{x=-1}=\left.\frac{\partial \mathrm{Gr}}{\partial x}\right|_{x=1}=0
$$

Of course $\mathrm{Gr}$ will be dependent on $\varepsilon$, but in the notation we have suppressed this dependence.

3.2.1. The 2 nd column of Gr. In order to construct the 2 nd column of Gr we put

$$
\left(\begin{array}{l}
J^{1} \\
J^{2}
\end{array}\right)=\left(\begin{array}{c}
0 \\
-\frac{1}{2}|t-x|
\end{array}\right)+\left(\begin{array}{l}
\tilde{J}^{1} \\
\tilde{J}^{2}
\end{array}\right) .
$$


Note, that the first term has the correct singularity at $x=t . \tilde{J}_{1}$ and $\tilde{J}^{2}$ will be continuously differentiable at $x=t$ and they are of the following form

$$
\begin{aligned}
\tilde{J}^{1}= & \left(J_{0}^{1}+\varepsilon D_{1}\right) H+\left(J_{0}^{1}+\varepsilon \bar{D}_{1}\right) \bar{H} \\
& +(1-H-\bar{H}) \cdot\left\{\varepsilon^{-1} E_{-1}+E_{0}\right\}
\end{aligned}
$$

(3.2.1.3) $\tilde{J}^{2}=J_{0}^{2} H+\bar{J}_{0}^{2} \bar{H}+(1-H-\bar{H})\left\{J_{0}^{2}(y)+\varepsilon \hat{E}_{1}+\varepsilon^{2} \hat{E}_{2}\right\}$.

Here $J_{0}^{1}, J_{0}^{2}, \bar{J}_{0}^{1}, \bar{J}_{0}^{2}$ play the role of the regular expansion which together with some constant $c$ will be found from a problem of type (2.2.1). The constant $c$ comes from the term $\varepsilon^{-1} E_{-1}$ in the internal layer, where $E_{-1}$ is just a solution of the homogeneous layer equation in (2.3.1.1).

$$
E_{-1}=-c T_{0}^{\prime} \text {. }
$$

Note, that the presence of the term $E_{-1}$ is typically a resonance phenomenon. The next term has to satisfy the equation

$$
\left[\frac{d^{2}}{d \xi^{2}}-\hat{f}_{u}^{0}(\xi)\right] E_{0}=-c\left(\xi \hat{f}_{u x}^{0}+T_{1} \hat{f}_{u u}+Y_{1} \hat{f}_{u v}^{0}\right) T_{0}^{\prime}+J_{0}^{2}(y) \cdot \hat{f}_{v}^{0}
$$

where the constant $c$ is chosen, such that the r.h.s. is orthogonal to $T_{0}^{\prime}$ in $L_{2}$-sense. The solution $E_{0}$ is then given by the expression in (2.3.1.6). In this case the value of the free constant multiplying $T_{0}^{\prime}$ in this solution is unimportant, we shall take that constant $=0$. Now $J_{0}^{1}, J_{0}^{2}, \bar{J}_{0}^{1}, \bar{J}_{0}^{2}$ and $c$ follow from

$$
\begin{aligned}
\mathscr{L}\left(J_{0}^{1}, J_{0}^{2}, \bar{J}_{0}^{1}, \bar{J}_{0}^{2}, c\right) & \\
= & \left(\frac{1}{2}|t-x| f_{v}^{0}, \frac{1}{2}|t-x| g_{v}^{0}, \frac{1}{2}|t-x| \bar{f}_{v}^{0}\right. \\
& \left.\frac{1}{2}|t-x| \bar{g}_{v}^{0} ; 0,0 ; 0,0 ; 0\right) .
\end{aligned}
$$

It is easy to check, that

$$
E_{0}(\xi) \underset{\xi \rightarrow \infty}{\rightarrow} J_{0}^{1}(y), \quad E_{0}(\xi) \underset{\xi \rightarrow \infty}{\rightarrow} \bar{J}_{0}^{1}(y) .
$$

where the convergence is of exponential type.

For the second component in the internal layer we obtain

$$
\frac{d^{2} \hat{E}_{1}}{d \xi^{2}}=-c T_{0}^{\prime} \cdot \hat{g}_{u}^{0}
$$




$$
\frac{d^{2} \hat{E}_{2}}{d \xi^{2}}=\hat{g}_{u}^{0} E_{0}+\hat{g}_{v}^{0} J_{0}^{1}(y)-c T_{0}^{\prime}\left\{\hat{g}_{u u}^{0} T_{1}+\hat{g}_{u v}^{0} y_{1}+\hat{g}_{u x}^{0} \xi\right\} \underset{\mathrm{def}}{=} \hat{w}_{0} .
$$

Using the matching conditions and choosing constants which are still free in a convenient way this leads to

$$
\begin{gathered}
(3.2 .1 .10) \quad \hat{E}_{1}=\left(J_{0}^{2}\right)^{\prime}(y) \cdot \xi-c \int_{-\infty}^{\xi}\left[g\left(y, T_{0}(\xi), 0\right)-g(y, 0,0)\right] d \xi \\
(3.2 .1 .11) \quad \hat{E}_{2}=\int_{0}^{\infty}(\xi-\bar{\xi}) \hat{w}_{0}(\bar{\xi}) d \bar{\xi} .
\end{gathered}
$$

A simple calculation shows, that the following overlap property holds:

$$
\begin{aligned}
& \left|J_{0}^{2}(x)-\left\{J_{0}^{2}(y)+\varepsilon \hat{E}_{1}+\varepsilon^{2} \hat{E}_{2}\right\}\right| \\
& \quad \leq C\left(|x-y|^{3}+\varepsilon+\varepsilon^{2}|\xi|\right) \text { for } y-2 \sigma \leq x \leq y-\sigma .
\end{aligned}
$$

Such an overlap is also present at the other side of the transition point. Moreover analogous estimates hold for the derivatives. Finally, $D_{1}$ and $\bar{D}_{1}$ are just corrections to satisfy the boundary codnitions

$$
\begin{aligned}
& D_{1}(\eta)=\frac{1}{a}\left(J_{0}^{1}\right)^{\prime}(-1) \cdot \exp (-a \eta) \\
& \bar{D}_{1}(\eta)=\frac{-1}{\bar{a}}\left(J_{0}^{1}\right)^{\prime}(1) \cdot \exp (-\bar{a} \bar{\eta}),
\end{aligned}
$$

compare (2.3.2.1).

We can now immediately verify, that the 2 nd column of $\mathrm{Gr}$ as defined above has properties as necessary in view of (i)-(iv). We find, that the 2 nd column of $\mathbf{A}$ is identically $\left(\begin{array}{l}0 \\ 0\end{array}\right)$, since the jump in the derivative is exactly correct, and we obtain

$$
\int_{-1}^{1}\left|“ L_{\varepsilon} \mathrm{Gr}_{, 2}\right| d t \leq K \varepsilon \ln \left(\frac{1}{\varepsilon}\right)
$$

in an obvious notation.

The verification of (3.2.1.14) is analogous to $\S(2.4)$ and we leave the details to the reader.

To conclude this section we want to point out, how remarkable it is, that in the construction of the 2nd column of $\mathrm{Gr}$ we don't have to distinguish between various cases for the location of $t$. For example, $t$ in the internal layer is no special case here, it simply introduces some $\varepsilon$-dependence in the "regular part" $J_{0}^{1}, J_{0}^{2}$, etc. but that is all. This will be different in the next section.

3.2.2. The 1st column of Gr. Here we distinguish between 3 regions for the location of $t$.
(a) $t \in[-1, y-\sigma]$,
(b) $t \in[y+\bar{\sigma}, 1]$,
(c) $t \in[y-2 \sigma, y+2 \bar{\sigma}]$. 
Let us call this column $I^{a}, I^{b}$ and $I^{c}$ in these respective cases. Then the 1st column $I$ for $(x, t) \in[-1,1]^{2}$ will be given by

$$
I=H_{2} I^{a}+\bar{H}_{2} I^{b}+\left(1-H_{2}-\bar{H}_{2}\right) I^{c}
$$

with $H_{2}=\chi((y-t) / \sigma), \bar{H}_{2}=\chi((t-y) / \bar{\sigma})$ with $\chi$ the cut-off function introduced in (2.3.5).

ad $a: I^{a}$ is introduced in the following way:

(3.2.2.2) $\quad I^{a}=(1-\bar{H})\left(\begin{array}{c}-\frac{1}{2 \varepsilon \lambda} \exp (-\lambda|\zeta|)+\varepsilon^{-1} M_{-1} \\ S_{0}+\varepsilon N_{1}\end{array}\right)+\left(\begin{array}{c}\tilde{I}^{1, a} \\ \tilde{I}^{2, a}\end{array}\right)$

Here $\lambda=\sqrt{f_{u}^{0}(t)}$ and $\zeta=(x-t) / \varepsilon$. It is clear, that the first term of the first component has exactly the correct jump in the derivative at $x=t$. To correct the error in the b.c. we take $M_{-1}$ as the following solution of the homogeneous boundary layer equation at $x=-1$ :

$$
M_{-1}(\eta)=-\frac{1}{2} \exp (-\lambda(1+t) / \varepsilon) \cdot a^{-1} \exp (-a \eta)
$$

with $a=\sqrt{f_{u}^{0}(-1)}$, compare (2.3.2.1).

$S_{0}$ is introduced to compensate the effect of $-(2 \varepsilon \lambda)^{-1} \exp (-\lambda|\zeta|)$ in the second equation

$$
S_{0}=\int_{x}^{y}\left(x-x^{\prime}\right) g_{u}^{0} \frac{e^{-\lambda\left|x^{\prime}-t\right| / \varepsilon}}{2 \varepsilon \lambda} d x^{\prime} .
$$

Note, that $S_{0}$ decays exponentially in $\zeta$ for $\zeta \rightarrow \infty$ and behaves as $(x-t) g_{u}^{0}(t)+$ exponentially small terms in $\zeta$ for $\zeta \rightarrow-\infty$.

In a similar way $N_{1}$ compensates the effect of $\varepsilon^{-1} M_{-1}$ :

$$
N_{1}=g_{u}^{0}(-1) a^{-2} M_{-1}
$$

The other terms in (3.2.2.2) will be of the following form

$$
\begin{aligned}
\tilde{I}^{1, a}= & {\left[I_{0}^{1}+\varepsilon M_{0}\right] H+\left[\bar{I}_{0}^{1}+\varepsilon \bar{M}_{0}\right] \bar{H} } \\
& +(1-H-\bar{H})\left\{-\varepsilon^{-1} A T_{0}^{\prime}+\Lambda_{0}\right\} \\
\tilde{I}^{2, a}= & I_{0}^{2} H+\bar{I}_{0}^{2} \bar{H}+(1-H-\bar{H})\left\{I_{0}^{2}(y)+\varepsilon \Omega_{1}+\varepsilon^{2} \Omega_{2}\right\} .
\end{aligned}
$$

Since the process to construct $I^{1, a}, I^{2, a}$ is so similar to $\S(3.2 .1)$, we only give a brief sketch.

Having the calculations of $\$ 3.2 .1$ in mind it will not be surprising, that $I_{0}^{1}, I_{0}^{2}, \bar{I}_{0}^{1}, \bar{I}_{0}^{2}$ and $A$ are the solution of an inhomogeneous problem 
as in 2.2.1:

$$
\begin{aligned}
& \mathscr{L}\left(I_{0}^{1}, I_{0}^{2}, \bar{I}_{0}^{1}, \bar{I}_{0}^{2}, A\right) \\
& =\left(f_{v}^{0} S_{0}, g_{v}^{0} S_{0}, 0,0 ;-S_{0}^{\prime}(-1)-\frac{d N_{1}}{d \eta}(0), 0 ; 0,0 ; 0\right) .
\end{aligned}
$$

Consequently the r.h.s. in the equation for $\Lambda_{0}$ is orthogonal to $T_{0}^{\prime}$ and $\Lambda_{0}$ is determined in the same way as $E_{0}$ in (3.2.1.5), but with $c$ replaced by $A$ and $J_{0}^{2}(y)$ replaced by $I_{0}^{2}(y)$. Of course, $\Omega_{1}, \Omega_{2}$ are found analogous to $\hat{E}_{1}, \hat{E}_{2}$ in (3.2.1.8-11) with the same changes $c \rightarrow A, J_{0}^{2} \rightarrow I_{0}^{2}$. Finally, the boundary layer corrections $M_{1}, \bar{M}_{1}$ are as in (3.2.1.13), but with $I_{0}^{1}$ instead of $J_{0}^{1}$. It will be clear, that estimates analogous to (3.2.1.8) and (3.2.1.12) hold in this case. Therefore we find for this part of the first column the correct properties in view of (i)-(iv), specifically

$$
\int_{-1}^{y}\left|“ L_{\varepsilon} \mathrm{Gr}_{0,1} "\right| d t \leq K \varepsilon \ln \left(\frac{1}{\varepsilon}\right) \text {. }
$$

$a d b$ : The construction of $I^{b}$ is completely analogous to the construction of $I^{a}$.

ad $c: I^{c}$ will be of the following form:

$$
I^{c}=\left(\begin{array}{c}
-\frac{1}{2 \varepsilon} \exp (-|\zeta|) \\
0
\end{array}\right)+\left(\begin{array}{c}
\tilde{I}^{1, c} \\
\tilde{I}^{2, c}
\end{array}\right)
$$

with $\zeta=(x-t) / \varepsilon$ as before and with $t=y+\varepsilon \tau, \zeta=\xi-\tau$. Further:

$$
\begin{aligned}
\tilde{I}^{1, c}= & \left(\varepsilon^{-1} I_{-1}^{1}+N_{0}\right) H+\left(\varepsilon^{-1} \bar{I}_{-1}^{1}+\bar{N}_{0}\right) \bar{H} \\
& +(1-H-\bar{H})\left\{-\varepsilon^{-2} B T_{0}^{\prime}+\varepsilon^{-1} \Lambda_{-1}\right\} \\
\tilde{I}^{2, c}= & \varepsilon^{-1} I_{-1}^{2} H+\varepsilon^{-1} \bar{I}_{-1}^{2} \bar{H} \\
& +(1-H-\bar{H})\left\{\varepsilon^{-1} I_{-1}^{2}(y)+\Omega_{0}+\varepsilon \Omega_{1}\right\} .
\end{aligned}
$$

It is not necessary to include more terms in the expansions here, since $I^{c}$ is only used for $t$ in an $O(\varepsilon \ln (1 / \varepsilon))$ interval, which yields an extra small factor in the integration in (3.2.4).

Furthermore, the structure of the first column of $\mathrm{Gr}$ with $t$ in the internal layer shows the resonance even more drastically then before, because of the term $-\varepsilon^{-2} B T_{0}^{\prime}$. 
In this case $\Lambda_{-1}$ has to satisfy the equation

$$
\begin{aligned}
(3.2 .2 .11)\left(\frac{d^{2}}{d \xi^{2}}-\hat{f}_{u}^{0}\right) \Lambda_{-1}= & -\left(\xi \hat{f}_{x u}^{0}+T_{1} \hat{f}_{u v}^{0}\right) B T_{0}^{\prime}+\hat{f}_{v}^{0} I_{-1}^{2}(y) \\
& +\frac{1}{2}\left(1-\hat{f}_{u}^{0}\right) e^{-|\xi|}
\end{aligned}
$$

hence an extra forcing term arises in the equation. We introduce the notation

$$
\delta_{0}(\tau)=\frac{1}{2} \int_{-\infty}^{\infty}\left(1-\hat{f}_{u}^{0}(\xi)\right) e^{-|\xi-\tau|} T_{0}^{\prime}(\xi) d \xi
$$

Note, that $\delta_{0}(\tau)$ vanishes exponentially for $\tau \rightarrow-\infty$ and $\tau \infty+\infty$. Now, we find $I_{-1}^{1}, I_{-1}^{2}, \bar{I}_{-1}^{1}, \bar{I}_{-1}^{2}, B$ as the solution of the following problem

$$
\text { (3.2.2.13) } \mathscr{L}\left(I_{-1}^{1}, I_{-1}^{2}, \bar{I}_{-1}^{1}, \bar{I}_{-1}^{2}, B\right)=(0,0,0,0 ; 0,0 ; 0,0 ; \delta(\tau)) \text {. }
$$

Hence, for large $|\tau|$ this solution vanishes exponentially.

The r.h.s. of (3.2.2.11) is now orthogonal to $T_{0}^{\prime}$ and the solution $\Lambda_{-1}$ is found as in (2.3.1.6), where the free constant can be taken to be 0 . The construction of the other terms in (3.2.2.10) is again analogous to section (3.2.1). The result is that $\Omega_{0}, \Omega_{1}$ and $N_{0}, \bar{N}_{0}$ are given by the expressions in $(3.2 .1 .10,11)$ and $(3.2 .1 .13)$, respectively, but with the obvious changes $c \rightarrow B, J_{0}^{2} \rightarrow I_{-1}^{2}$. Further, mutatis mutandis (3.2.1.7) still holds and as an analogue of the overlap property in (3.2.1.12) we now have:

$$
\begin{aligned}
& \left|\varepsilon^{-1} I_{-1}^{2}(x)-\left\{\varepsilon^{-1} I_{-1}^{2}(y)+\Omega_{0}+\varepsilon \Omega_{1}\right\}\right| \\
& \quad \leq C \delta(\tau) \cdot\left\{\varepsilon^{-1}(x-y)^{3}+1+\varepsilon^{2}|\xi|\right\}
\end{aligned}
$$

in the region $y-2 \sigma \leq x \leq y-\sigma$.

Putting all things together we find that the contribution of $I^{c}$ has the correct properties in view of (i)-(iv). Especially we mention that

$$
\int_{y-2 \sigma}^{y+2 \bar{\sigma}} \mid \text { “ } L_{\varepsilon} \mathrm{Gr}_{,, 1} " \mid d t \leq K \varepsilon \ln \left(\frac{1}{\varepsilon}\right) \text {. }
$$

Herewith the construction of the approximate Green matrix is complete.

3.3. The approximate inverse operator and the derivation of the correctness result. Using the properties of $\mathrm{Gr}$ given in (i)-(iv) it follows, that $\left(L_{\varepsilon}^{-1}\right)^{\prime}$ as defined in (3.1.18) is an operator with domain $\{C[-1,1]\}^{2}$ and range contained in the domain of $L_{\varepsilon}$. Moreover

$$
L_{\varepsilon}\left(L_{\varepsilon}^{-1}\right)^{\prime}=I+\varepsilon^{\nu} K_{\varepsilon}
$$

where actually $K_{\varepsilon}$ is purely an integral operator with kernel “ $L_{\varepsilon} \mathrm{Gr}$ ”, since we succeeded to do the construction with $\mathbf{A}$ in (3.2.3) $\equiv 0$. We take as the Banach space $B$ in $\$ 3.1, B=\{C[-1,1]\}^{2}$ provided with the maximum 
norm ||$_{0}$ being the sum of the norms ||$_{\max }$ for each of the components.

Of course (3.2.4) implies, that the operator $\varepsilon^{\nu} K_{\varepsilon}$ satisfies the estimate in (3.1.8):

$$
\left|\varepsilon^{\nu} K_{\varepsilon}\right|_{0} \leq K \varepsilon^{\nu}
$$

Using explicitly the form of Gr given in $§ 3.2$ it is possible to estimate the norm of $\left(L_{\varepsilon}^{-1}\right)^{\prime}$ as

$$
\left|\left(L_{\varepsilon}^{-1}\right)^{\prime}\right|_{0} \leq m \varepsilon^{-1}
$$

With an $\varepsilon$-independent constant $m>0$. The factor $\varepsilon^{-1}$ in this norm is due to a resonance effect in the internal layer, which is only present in the first component. It is left to the reader to check, that this resonance effect is precisely what one should expect on the basis of the construction of a formal approximation for the linearised problem with smooth inhomogeneous terms.

In this case the non-linear operator $N_{\varepsilon}$ is given by

$$
N_{\varepsilon}\left(\begin{array}{l}
v_{1} \\
v_{2}
\end{array}\right)=\int_{0}^{1}(1-z) \frac{d^{2}}{d z^{2}}\left\{\left(\begin{array}{l}
f \\
g
\end{array}\right)\left(x, Z_{1}^{N}+z v_{1}, Z_{2}^{N}+z v_{2}\right)\right\} \cdot d z .
$$

Using a method as in [7], [8] it is easy to show, that

$$
\left|N\left(\begin{array}{l}
v_{1} \\
v_{2}
\end{array}\right)-N\left(\begin{array}{l}
w_{1} \\
w_{2}
\end{array}\right)\right|_{0} \leq l_{0} \rho\left|\left(\begin{array}{l}
v_{1} \\
v_{2}
\end{array}\right)-\left(\begin{array}{l}
w_{1} \\
w_{2}
\end{array}\right)\right|_{0}
$$

for $0 \leq \rho \leq \rho_{2}$ and $0<\varepsilon \leq \varepsilon_{2}$ with $\varepsilon$-independent positive constants $l_{0}$ and $\rho_{2}$. In combination with (3.3.3) this leads to the following value for $\nu(\varepsilon)$ in (3.1.12).

$$
\nu(\varepsilon)=\varepsilon\left(l_{0} m\right)^{-1} .
$$

Now an application of the results in (3.1.15-16) show that there is a unique solution of (1.1-2) satisfying the estimate

$$
\left|\left(\begin{array}{l}
u \\
v
\end{array}\right)-\left(\begin{array}{c}
Z_{1}^{N} \\
Z_{2}^{N}
\end{array}\right)\right|_{0} \leq 4 m \varepsilon^{-1} \cdot \tilde{R}_{N}\left[\varepsilon \ln \left(\frac{1}{\varepsilon}\right)\right]^{N+1}
$$

for $N \geq 2$.

The main result given in (3.1) is then an easy consequence of (3.3.7) and the triangle inequality

$$
\left|\left(\begin{array}{l}
u \\
v
\end{array}\right)-\left(\begin{array}{c}
Z_{1}^{N} \\
Z_{2}^{N}
\end{array}\right)\right|_{0} \leq\left|\left(\begin{array}{c}
u \\
v
\end{array}\right)-\left(\begin{array}{c}
Z_{1}^{N+2} \\
Z_{2}^{N+2}
\end{array}\right)\right|_{0}+\left|\left(\begin{array}{c}
Z_{1}^{N+2} \\
Z_{2}^{N+2}
\end{array}\right)-\left(\begin{array}{c}
Z_{1}^{N} \\
Z_{2}^{N}
\end{array}\right)\right|_{0}
$$

The latter term is $O\left(\varepsilon^{N+1}\right)$ because of the structure of the formal approximation. 


\section{REFERENCES}

[1] M. S. Berger and L. E. Fraenkel, On singular perturbations of non-linear operator equations, Indiana Univ. Math. J., 20, (1971).

[2] C. M. Brauner, W. Eckhaus, M. Garbey, and A. van Harten, Asymptotics with unusual order functions in a free surface problem, preprint, M.I., R.U.U., (1984).

[3] W. Eckhaus, Matched Asymptotic Expansions, North-Holland, Amsterdam, (1978).

[4] P. C. Fife, Boundary and interior transition layer phenomena for pairs of 2 nd order diff. eq., J. Math. Anal. Appl., 54 (1976).

[5] _ Mathematical Aspects of Reacting and Diffusing Systems, Lecture Notes in Biomath., 28 (1979), Springer, Berlin.

[6] H. Fujii, M. Mimura, and Y. Nishiura, A picture of the global bifurcation diagram in ecological interacting and diffusing systems, Physica D-Nonl. Phenomena, 5D, (1982).

[7] A. van Harten, Singularly perturbed non-linear 2 nd order elliptic boundary value problems, thesis, University of Utrecht, 1975.

[8] _ Non-linear singular perturbation problems: proofs of correctness of a formal approximation based on a contraction principle in a Banach space, J. Math. Anal. Appl., 65 (1978).

[9] Y. Hosono and M. Mimura, Singular perturbations for pairs of two-point boundary value problems of Neumann type, Lecture Notes in Numer. Appl. Anal., 2 (1980).

[10] Y. Nishiura, Global structure of bifurcating solutions of some reaction diffusion systems and their stability problem, 5th Int. Symp. on Comp. Meth. in Appl. Sc. and Eng., Versailles, (1981).

[11] R. S. Smith, A Green function for a singularly perturbed Dirichlet problem, preprint, University of California, San Diego, (1984).

[12] W. Wasow, Singular perturbations of boundary value problems for non-linear differential equations of 2 nd order, Comm. Pure Appl. Math., 9 (1956).

[13] J. Yarmish, Newton's method techniques for singular perturbations, SIAM J. Math. Anal., 6 (1975).

Received January 2, 1985.

UNIVERSITY OF UTRECHT

3508 TA UTRECHT, THE NETHERLANDS 



\section{PACIFIC JOURNAL OF MATHEMATICS EDITORS}

\author{
V. S. VARADARAJAN \\ (Managing Editor) \\ University of California \\ Los Angeles, CA 90024 \\ HERBERT ClEMENS \\ University of Utah \\ Salt Lake City, UT 84112 \\ R. FINN \\ Stanford University \\ Stanford, CA 94305
}

\author{
HERMANN FLASCHKA \\ University of Arizona \\ Tucson, AZ 85721 \\ RAMESH A. GANGOLLI \\ University of Washington \\ Seattle, WA 98195 \\ VAUghan F. R. JONES \\ University of California \\ Berkeley, CA 94720 \\ ROBION KIRBY \\ University of California \\ Berkeley, CA 94720
}

C. C. MOORE

University of California Berkeley, CA 94720

H. SAMELSON

Stanford University Stanford, CA 94305

HAROLD STARK

University of California, San Diego La Jolla, CA 92093

\section{ASSOCIATE EDITORS}
R. ARENS
E. F. BECKENBACH
B. H. NEUMANN
F. WOLF
K. YOSHIDA (1906-1982)

\section{SUPPORTING INSTITUTIONS}

UNIVERSITY OF ARIZONA

UNIVERSITY OF BRITISH COLUMBIA

CALIFORNIA INSTITUTE OF TECHNOLOGY

UNIVERSITY OF CALIFORNIA

MONTANA STATE UNIVERSITY

UNIVERSITY OF NEVADA, RENO

NEW MEXICO STATE UNIVERSITY

OREGON STATE UNIVERSITY
UNIVERSITY OF OREGON UNIVERSITY OF SOUTHERN CALIFORNIA

STANFORD UNIVERSITY

UNIVERSITY OF HAWAII

UNIVERSITY OF TOKYO

UNIVERSITY OF UTAH

WASHINGTON STATE UNIVERSITY

UNIVERSITY OF WASHINGTON 


\section{Pacific Journal of Mathematics}

Vol. 125, No. 1 September, 1986

Gilles Christol, Fonctions et éléments algébriques $\ldots \ldots \ldots \ldots \ldots \ldots \ldots \ldots$

Jo-Ann Deborah Cohen, Extensions of valuation and absolute valued

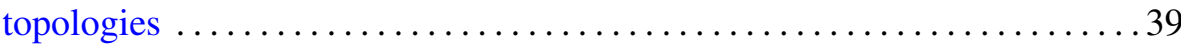

Miriam Cohen, Smash products, inner actions and quotient rings . . . . . . 45

Mikio Furushima, On the singular $K-3$ surfaces with hypersurface

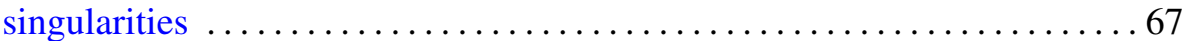

Gerhard Gierz and Boris Shekhtman, A duality principle for rational

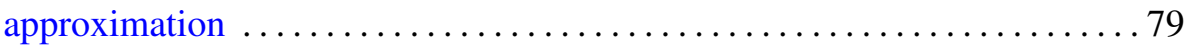

Anthony Wood Hager, A description of HSP-like classes, and

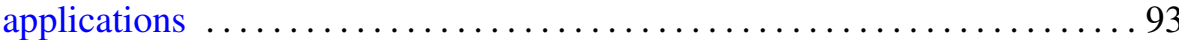

George Alan Jennings, Lines having high contact with a projective

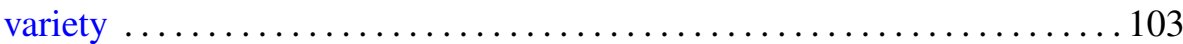

John Lott, Eigenvalue bounds for the Dirac operator . . . . . . . . . . . 117

Denis Laurent Luminet, A functional calculus for Banach PI-algebras . . . . 127

Shizuo Miyajima and Noboru Okazawa, Generators of positive

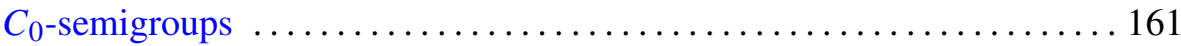

Takemi Mizokami, On functions and stratifiable $\mu$-spaces $\ldots \ldots \ldots \ldots \ldots 177$

Jeff Parker, 4-dimensional $G$-manifolds with 3-dimensional orbits . . . . . 187

Elias Saab and Paulette Saab, On Peł czyński's properties (V) and (V*) . . 205

Elmar Schrohe, The symbols of an algebra of pseudodifferential operators

Aart van Harten and Els Vader-Burger, Approximate Green functions as a tool to prove correctness of a formal approximation in a model of competing and diffusing species

Stephen Watson, Using prediction principles to construct ordered

continua 